\title{
Hacia una etnografía de la lectura escolar. Prácticas y representaciones de la lectura en la escuela'
}

\author{
Towards an Ethnography of Reading at School. \\ Practice and Representations of Reading at school
}

\author{
AURORA MARÍA RUIZ-BEJARANO \\ Universidad de Cádiz \\ España \\ auroramaria.ruiz@uca.es
}

(Recibido: I4-O3-20I5; aceptado: o6-OI-2OI6)

\begin{abstract}
Resumen. En los últimos años han surgido en nuestro país estudios que, pese a su dispersión disciplinar, epistemológica y metodológica, contemplan la lectura como un fenómeno social y cultural situado. En el ámbito educativo, la ausencia de una tradición metodológica empuja a buscar en la etnografía las herramientas que permitan investigar el entramado de la lectura de una escuela desde el análisis de sus prácticas, sus representaciones, sus espacios y sus discursos.

El propósito de este artículo es analizar ese proceso de creación metodológica a través del estudio de un caso.
\end{abstract}

Palabras clave: literacidad; etnografia; investigación educativa; lectura; estudio de caso.
Abstract. In recent years there have been several studies, from different disciplines, which have examined the reading process as a social and cultural phenomenon. However, in the Spanish educational field, the literacy studies have not shown a strong ethnographic tradition.

The purpose of this article is to provide some methodological tools for the analysis of practices and representations of reading at school through a case study.

Keywords: literacy, ethnography; educational research; reading; case study.

\footnotetext{
${ }^{\text {I }}$ Para citar este artículo: Ruiz-Bejarano, Aurora María (20I6). Hacia una etnografía de la lectura escolar. Prácticas y representaciones de la lectura en la escuela. Alabe 14. [www.revistaalabe.com] DOI: IO.I5645/Alabe20I6.I4.2
} 


\section{Los desafíos de una etnografía de la lectura escolar}

Hace apenas unos años que han ido surgiendo en nuestro país estudios que, pese a su dispersión disciplinar, epistemológica y metodológica, comparten la visión de la lectura como un fenómeno social y cultural situado (Cassany, 2006; Martos García, 2OIO). De forma generalizada, estos trabajos se han reunido bajo el paraguas de los estudios culturales de la lectura, de los nuevos estudios sobre alfabetismos o de los llamados Nuevos Estudios de Literacidad (NEL). De uno u otro modo, todos estos trabajos se distancian de los enfoques cognitivo y lingüístico predominantes en las investigaciones acerca de la lectura y se reconocen deudores de las investigaciones pioneras de Roger Chartier (I994) sobre la historia sociocultural de las prácticas de la lectura y de las formas de sociabilidad asociadas a ellas. Más allá de estas similitudes, poco conservan en común².

Dentro de este conjunto de trabajos agrupados se situaba la investigación de mi tesis doctoral, Prácticas y representaciones de la lectura en la escuela. Estudio de un caso. El proyecto fue estructurado con el propósito de analizar y comprender qué sentidos atribuía una comunidad educativa a la lectura en la escuela desde el análisis de las prácticas promovidas dentro de esta institución, de los discursos producidos sobre la lectura y de los espacios donde se generan.

Todos estos focos de indagación (el estudio de los eventos letrados y de las prácticas de lectura en la escuela, el examen de los espacios físicos de lectura, de los espacios letrados y el análisis de los discursos sobre esta) habrían de conducir a la reconstrucción del entramado de la lectura producido en el seno de la comunidad de un centro de Educación Infantil y Primaria específico, pues, haciendo propias las palabras de Díaz de Rada "la etnografía no surge de la adición de materiales empíricos producidos con diferentes técnicas, sino de su articulación en una trama de intenciones teóricas y metodológicas” (2OI2: I5).

Establecido bajo esos parámetros, el proyecto se perfilaba como una investigación de corte etnográfico con estudio de caso. En ese punto inicial donde se decide el diseño metodológico, surgió un primer escollo que implicaba, a su vez, uno de los desafíos del proceso investigador. Un escollo en la medida en que la dispersión teórica dificultaba el hallazgo de patrones que orientasen el trabajo de campo. Al mismo tiempo, suponía

\footnotetext{
${ }^{2}$ Como muestra de esta dispersión, dentro de nuestro ámbito nacional se pueden señalar algunos trabajos y trayectorias investigadoras destacadas desde el ámbito de la Lingüística, la Literatura o la Historia de la Educación. Desde la Universidad Complutense de Madrid destaca, por citar un primer ejemplo, el trabajo de María del Carmen González Landa (2009) sobre la lectura y la escritura como procesos interactivos y situados. Por otro lado, en la Universitat Pompeu Fabra de Barcelona, Daniel Cassany dirige desde el año 2004 el Grupo de Investigación sobre Literacitat Crítica, centrado en explorar prácticas letradas dentro de entornos digitales y mediáticos. A su vez, desde la Universidad de Extremadura, Eloy Martos y Agustín Vivas profundizan a través de sus estudios en las artes performativas contemporáneas "que se sirven de la palabra como argamasa o al menos hilo conductor" (2OIO: 6), como es el caso de los cuenta-cuentos, de las artes comediográficas o de los recitales poéticos. En las antípodas de estos tres grupos de investigaciones y dentro del ámbito educativo son relevantes las incursiones realizadas por Viñao Frago (I999, 200I) en el campo de la historia de la alfabetización escolar.
} 
un desafío en cuanto a que la imprecisión metodológica asociada a la ausencia de una tradición teórica consolidada, empujaba a buscar formas alternativas para encauzar la investigación al mismo tiempo que esta se iba desarrollando. Porque, ¿cómo se reconstruye el entramado de la lectura de una escuela?, ¿cuáles son sus elementos componentes y, en consecuencia, sobre qué aspectos se debe indagar? En definitiva, ¿qué significa realizar una etnografía de la lectura escolar?, ¿cómo se afronta su planificación y posterior desarrollo?

Estas dos últimas cuestiones (el alcance de una aproximación ecológica a la lectura en la escuela y el trayecto metodológico a seguir en este acercamiento) se emplazaron pronto como las dos primeras a afrontar dentro de la investigación. Y ambas se postulan ahora como las guías de este artículo.

El objetivo de estas páginas es realizar un repaso al proceso seguido en la construcción de un marco metodológico que permitiese la aproximación a la lectura en la escuela como un fenómeno social y cultural situado (Barton y Hamilton, I998).

Más allá de este objetivo, el propósito último de este artículo arraiga en la propia naturaleza de la investigación etnográfica: su carácter indefectiblemente idiográfico que lo aleja, por oposición, de cualquier pretensión nomotética. Este recurso narrativo de la propia experiencia investigadora, desde la irrepetible singularidad de su contingencia, pretende contribuir con comedimiento al debate teórico, epistemológico y metodológico acerca de las posibilidades de una aproximación situada de las prácticas y representaciones de la lectura en la escuela.

Las reflexiones que re recogen se centran en una parte de la experiencia del trabajo de campo; aquella relacionada con la recogida de la información y estrategias metodológicas utilizadas en las incursiones a la escuela María Moliner 3 . Concretamente, son dos las cuestiones sobre las que se articula el contenido del capítulo:

- ¿Qué estudiar en una aproximación situacional de la lectura en la escuela?, ¿qué ámbitos de indagación se pueden delimitar?

• ¿Cómo proceder durante la investigación?; es decir, ¿a qué estrategias metodológicas es posible recurrir?

3 Nombre ficticio dado al centro de Educación Infantil y Primaria de la ciudad de Sevilla donde se desarrolló el estudio de caso con el fin de preservar su anonimato. 


\section{Prácticas y representaciones de la lectura en la escuela: la selección de los ámbitos de investigación}

Sobre los límites que definen la etnografía de la lectura, escribe Martos García que esta:

se plantea interrogantes sobre los usos individuales y colectivos de la lectura, así como sobre las representaciones e identidades que formulan los lectores sobre el leer y el escribir. Del mismo modo, una investigación etnográfica tiene que describir los lugares físicos, escenarios y «rituales» (20IO: 200).

En efecto, una etnografía de la lectura se pregunta acerca del uso que los sujetos hacen de la lectura, de las representaciones construidas y de las identidades que se producen y que entran en juego: ¿por qué leen los individuos?, ¿qué leen asiduamente (blogs, novelas, cómics,...)?, ¿qué múltiples significados adquiere la lectura?... Asimismo, se interroga acerca de los escenarios en los que se produce la lectura, pero sin descuidar las formas en que se desarrolla dentro de esas fronteras físicas, es decir, aproximándose, como señala la cita anterior, a los ritos de la lectura: ¿dónde se lee (en la biblioteca, en una cafetería, en un taller literario, en el parque, en el dormitorio propio)? y ¿cómo se produce la lectura en cada uno de estos espacios? Se trata, en definitiva, de conocer la lectura desde el núcleo de su propia acción.

Todas estas cuestiones son, sin duda, una mera ilustración dentro de un vasto universo de posibles que no se agota en los ejemplos propuestos. Etnografías de la lectura dispares, con objetivos diferentes, formularán cuestiones que no necesariamente son las anteriormente expuestas como ejemplo. Sin embargo, grosso modo, tales interrogantes rondarán los términos antes indicados.

Existe una cierta similitud entre las cuestiones elaboradas por Martos García y las que se pueden plantear en una etnografía de la lectura escolar. Pero se trata de unas similitudes limitadas, pues lo cierto es que dichas cuestiones, al plantearse dentro del marco de una escuela, deben ser reconducidas y enfocadas desde una óptica, hasta cierto punto, diferente. Incluso, es necesario añadir un tercer ámbito de investigación que puede denominarse el entorno relacional inmediato de la lectura o, también, sus entornos de proximidad (Martos Núñez, 20I3).

Las próximas páginas constituyen un recorrido analítico a través de estos tres ámbitos de indagación. Dos de estos focos se fundamentan en aquellos otros propuestos en la definición de Martos García (2OIO), aunque centrados ahora en el campo educativo. El tercero de los ámbitos se refiere al entorno relacional inmediato de la lectura:

- Usos, representaciones e identidades: los eventos letrados

- Los espacios físicos y sus ritos: los espacios letrados

- El entorno relacional inmediato 


\section{I. Los usos, las representaciones y las identidades}

Las cuestiones englobadas dentro del ámbito de los usos de la lectura, sus representaciones y las identidades producidas sugieren un primer dilema en su formulación al trasladarlas al terreno educativo. En el caso de la escuela, estas preguntas no necesariamente se dirigen a un sujeto al que se le invita a través de la entrevista etnográfica a desgranar los motivos que le llevan a leer o a no hacerlo. Es decir, la cuestión clave no se puede establecer en los términos anteriormente descritos (¿por qué leer, para qué leer?), y dirigirlos hacia el propio interlocutor. Más bien, las preguntas deben ser estas otras: ¿por qué leer en la escuela?, ¿para qué leer en su interior? O lo que es lo mismo, la dirección señala no hacia los propios sujetos entrevistados, miembros del equipo docente, sino a un tercer grupo de personas: los alumnos y alumnas de la escuela. La cuestión central es, por lo tanto, ¿por qué la escuela -una escuela particular y concreta y en este caso, el centro de Educación Infantil y Primaria María Moliner-, se enfrasca en desplegar una compleja red de actividades, talleres, concursos, tertulias, etc. en torno a la lectura, así como en crear espacios apropiados para su aprendizaje?; ¿por qué ese esfuerzo compartido desde la comunidad educativa por crear buenos lectores? Y, también, por qué no, ¿qué o quién es un buen lector o una buena lectora?

Dentro de este terreno, hay una breve cita que condensa lo que supone ahondar en estas cuestiones:

La verdad es que puesto que no sabemos lo que es un lector, menos aún podemos tener una noción exacta de lo que es un buen lector. ¿A qué llamamos, como profesores, un buen lector? (Moreno, 2005:I54)

Aunque pudiera parecerlo, el asunto que plantea Moreno en estas líneas no es cosa baladí. En el curso de las entrevistas etnográficas efectuadas en el proceso investigador al profesorado de la escuela María Moliner, formular una pregunta aparentemente sencilla, como ¿quién es o qué es un buen lector o una buena lectora? o también, ¿por qué o para leer? a menudo devolvía como respuesta un silencio, un titubeo o una mirada desconcertante y perpleja. Las cuestiones eran legítimas, pues el propósito de la investigación era conocido; más aún, la enunciación de las preguntas se producía en términos simples y, por tanto, resultaban comprensibles para el interlocutor y no dejaba lugar a dudas; 0 , al menos, eso era lo esperado.

La primera de las cuestiones, aquella referida a ¿quién es un buen lector?, ¿qué rasgos identifican a los buenos lectores? resultaba bien definida y no admitía una doble interpretación por parte del sujeto entrevistado. La dificultad que afrontaba la persona entrevistada mediante el silencio, la vacilación en la respuesta y los rodeos en la conversación no se hallaba, pues, en una deficiente concreción de la pregunta.

Quizás la segunda de las cuestiones, de no incluir los matices pertinentes, podría antojarse ambigua. Así, ante la pregunta, ¿por qué leer?, la respuesta de los sujetos entrevistados se deslizaba entre dos extremos. Por una parte, la razón de la lectura encuentra sus fundamentos en el ámbito de lo volitivo y de las decisiones personales. A pesar de las 
sesiones programadas de lecturas colectivas o individuales en el aula o en la biblioteca escolar, es en última instancia el alumno y la alumna quien elegirá en su tiempo de ocio entre leer o no hacerlo y quien, en el primero de los casos, tomará la decisión de qué leer (un cómic, una novela, un blog, etc.). Este hecho, cabe puntualizar, no excluye la preocupación de la escuela por garantizar la lectura frecuente entre el alumnado y que se materializa en prácticas como las anteriormente señaladas. Y es ahí hacia donde apuntaba realmente el sentido de la pregunta: ¿cuáles son los fundamentos de la razón de leer?, o también, ¿por qué la escuela despliega toda una red de prácticas que pivotan sobre el universo del libro y de la lectura con el fin de formar buenos lectores?

La primera de las interpretaciones de la cuestión referida lastraba un comprensible silencio perplejo: ¿cómo se puede preguntar acerca de las motivaciones personales que conducen a otra persona a aproximarse a la lectura o, para, en caso contrario, decidir no hacerlo? Sin embargo, el silencio que acompañaba a la segunda de las interpretaciones -aquella que apuntaba a los fundamentos de la lectura en la escuela- tenía una naturaleza distinta, más próxima a la reacción mostrada por los sujetos entrevistados al preguntarles acerca de qué es o quién es un buen lector o una buena lectora. ¿Qué sucedía en estos dos casos?, ¿dónde tenía lugar, entonces, el escollo? La traba debía hallarse en otro nivel, más allá del plano lingüístico o del comunicativo.

Algunas de las claves que ayudan a comprender este difícil punto al que abocaron algunas de las entrevistas etnográficas realizadas en la escuela María Moliner pueden sustraerse de la presentación que Gimeno Sacristán hace de su libro El alumno como invención. A propósito de la escuela, de sus protagonistas y sus relaciones, de sus inanimados componentes, de sus acciones y sus discursos, de sus prácticas y sus ritos, el autor, escribe:

Todo lo que nos es familiar tiende a ser apreciado como natural; cuando eso ocurre le damos carta de naturaleza a lo que nos rodea, a los contactos y relaciones que mantenemos con lo que nos circunda, como si su existencia fuera resultado de la espontaneidad, como si siempre hubiera existido e inevitablemente tuviera que existir. Esa habituación cala en nuestras vidas, da sentido a cómo entendemos y nos representamos el mundo de la cotidianidad; es decir, da contenido a nuestro sentido común (2OO3:I3).

La lectura, o en este caso particular, la lectura en la escuela, no es ajena a la fuerza de la naturalización a la que se refieren las palabras de Gimeno Sacristán. Las actividades de animación a la lectura diseñadas una mañana en el aula, las tertulias literarias que se organizan en el salón de actos y a las que es invitado algún conocido escritor de la literatura juvenil, la planificación de la revista escolar, un cuestionario que se entrega a las familias sobre costumbres y hábitos relacionados con la lectura en el hogar... todas estas actuaciones vinculadas a la lectura escolar se sustentan sobre un complejo universo representacional y simbólico compartido desde su riqueza, disparidad y heterogeneidad por la comunidad escolar. Un complejo universo que, en un movimiento de mutua reciprocidad, se alimenta de la propia praxis a la que ofrece sentido y que en definitiva, 
conforma la argamasa que aporta consistencia a nuestro sentido común y a los discursos acerca de la lectura escolar.

El silencio y la perplejidad de los sujetos entrevistados se explica ante el hecho de que cuestiones como qué es un buen lector o una buena lectora o por qué se ha de leer en la escuela, surgen ante ellos como un sinsentido que se enfrenta a la lógica y al sentido común que la naturalización constituye.

¿Qué alternativas se abren para salvar estos obstáculos?, ¿cómo indagar en las producciones de sentido de la lectura cuando se han solidificado y la naturalización las hace un bloque compacto aparentemente uniforme? Hay, al menos, dos medidas que se pueden adoptar para atajar esta problemática.

En primer lugar, seleccionar adecuadamente las preguntas de las entrevistas se postula como un paso decisivo que marca la diferencia entre una entrevista rica en matices y otra estancada y yerma.

Igualmente, y en segundo lugar, el recurso de otras estrategias metodológicas de recogida de la información como la observación etnográfica, las conversaciones informales y el análisis de documentos se presentan como herramientas valiosas, en la medida en que arrojan luz sobre nuevos ámbitos de investigación desde los que reorientar y enfocar las entrevistas etnográficas a fin de obtener información de mayor riqueza testimonial.

\subsection{De los espacios físicos a los espacios letrados: la lectura desde la acción}

En los últimos años, desde las investigaciones situadas dentro de los Nuevos Estudios de Literacidad, se ha venido empleando un nuevo concepto: los espacios letrados. Con su uso se hace un mayor énfasis en la aproximación cultural de la lectura, señalando hacia su interpretación como conjunto de prácticas sociales que se captan desde la propia acción en la que suceden, desde la interacción de los sujetos involucrados en ellas y desde las formas de sociabilidad que producen. Este hecho implica un cierto distanciamiento del examen de los espacios de lectura desprovistos de las interacciones entre individuos que se desarrollan en su interior.

Tendemos a clasificar los espacios por las cosas o artefactos que hay dentro (biblioteca, mediateca, pinacoteca o museo, etc.), pero en este caso sería más coherente clasificarlos por las prácticas y procesos que se llevan a cabo en su interior. En efecto, un espacio letrado no es sólo un receptáculo sino un lugar que, por su propia naturaleza, invita a que ocurran cosas, y que, además, suele estar ligado a nuevas formas de relacionarse, de sociabilidad (Martos Núñez y Martos García, 2OІ2: ıІ6).

La descripción de un sencillo ejemplo extraído del estudio de caso en la escuela María Moliner ilustra la dicotomía existente entre el análisis de los espacios vacíos y el examen de los espacios letrados.

En la fecha del acceso al escenario de campo, una antigua aula de la planta baja, la número siete, alojaba la sala de la biblioteca escolar. Había estado ahí los últimos diez años. Se trataba de una habitación amplia, de paredes jalonadas en su parte más alta de 
láminas de vivos colores que recordaban las letras del alfabeto y el imperativo de respetar el silencio en la sala. Bajo las láminas, había estanterías repletas de libros que se sucedían unas a otras. Solo una de sus paredes estaba exenta de mobiliario. A lo largo de ella se distribuían los ventanales que traían luz desde el patio de recreo situado al otro lado de la frontera acristalada. Junto a una de las ventanas, un caballete sostenía una cartulina celeste donde un grupo de escolares había dibujado un esquema de los tejuelos de los libros con sus leyendas correspondientes: el azul acompañaba las obras reservados al Primer Ciclo de Educación Primaria, el amarillo para aquellas otras correspondientes al Segundo Ciclo y el rojo señalaba los libros recomendados al alumnado de Tercer Ciclo. En el extremo opuesto del ventanal, el escritorio del bibliotecario flanqueaba la puerta de entrada a la biblioteca. Sobre su tablero de melamina había un ordenador desvencijado, una impresora y algunos lápices y bolígrafos.

Junto a las estanterías, el principal mobiliario de la biblioteca lo constituía su conjunto de mesas anchas. Cuatro sillas se repartían en cada una de ellas, distribuidas en parejas a cada largo de la mesa, de forma que las unas quedaban enfrentadas a las otras y facilitaban la interacción de los usuarios de la biblioteca.

Visto así, la descripción física de esta sala, desprovista de sujetos interactuando ente sí, no ofrece información relevante acerca de los usos del espacio o de las prácticas de la lectura que se producían en ella; no permite analizar y comprender la naturaleza de los eventos letrados:

el concepto de “evento letrado" o prácticas de lectura aporta una visión más holística de los procesos de alfabetización, pues se insiste en el papel de lo interactivo y de la participación como ejes de descripción. Así, un lector aislado, un libro o una sala de biblioteca no conforman un evento de lectura, se precisa la interacción entre participantes, ámbito o contexto físico, artefactos y actividades (Martos García, 2OIO: 203).

Es cierto que durante el proceso investigador se podría haber realizado un análisis cuantitativo del inventario de volúmenes que se almacenan en sus estanterías. Se podría, incluso, haber accedido a la base de datos del ordenador de la sala y consultar el número de préstamos realizados en el último trimestre, el tipo de libro extraído y el perfil del alumnado que se había servido del servicio de préstamos de la biblioteca. Pero esa información no hubiese facilitado una mayor aproximación al objetivo de la investigación, a la recreación del entramado de la lectura de esta escuela.

Por otro lado, haber cesado la indagación en este punto y extraído conclusiones de la observación de este espacio desnudo de interacciones sociales, hubiese conducido a errores significativos en la investigación. ¿En qué sentido se hubiesen producido dichos errores? La continuación del ejemplo anterior lo aclara.

En el frontal de la puerta que da acceso a la sala había adherido un horario semanal. En él se distribuían las horas que se asignaban a cada grupo-clase para realizar lecturas colectivas en la biblioteca. De la observación del espacio se hubiese podido concluir fácilmente que la disposición de las mesas, con las sillas a su alrededor, era síntoma de las lecturas 
compartidas e interactivas que se realizaban cada semana. Pero el análisis de las sesiones de lectura colectiva, es decir, la observación sistemática de la lectura desde su acción hubiese mostrado el error de esas conclusiones.

Durante el período del trabajo de campo fue posible realizar algunas observaciones de sesiones de lectura colectiva de uno de los grupos de quinto curso de Educación Primaria. Los alumnos y las alumnas se repartían en las mesas, abrían el libro (el mismo título para toda la clase) y seguían silenciosos la lectura en voz alta que hacía el lector señalado por el dedo índice de la tutora. Aleatoriamente y sin aviso previo, se nombraba a un nuevo escolar que debía continuar la narración en el punto donde se había detenido su antecesor. Las distracciones y los leves murmullos se corregían y sancionaban en el acto, bien se tratase de una cabeza que se erguía para observar un pájaro tras la ventana, o de una alumna que indicaba a su compañero, despistado, el lugar por donde transcurría la lectura. Unas veces, al concluir, las preguntas acerca de lo leído se sucedían siguiendo el orden de las manos alzadas. En otras ocasiones, los escolares tomaban bolígrafos y cuadernos y daba comienzo un breve examen con el fin de evaluar el grado de comprensión de la lectura del día.

Del examen físico de la biblioteca no se hubiese podido elaborar una imagen de las prácticas de la lectura o de los eventos letrados tal y como se obtuvo posteriormente de las observaciones de las sesiones de lectura colectiva. Ni siquiera hubiese sido imaginable, porque, ¿cuál es el motivo que lleva a realizar estas sesiones colectivas en la biblioteca escolar? Este tipo de prácticas podían haberse llevado a cabo en el aula de referencia, donde los pupitres, a diferencia de lo que sucede en la biblioteca, se alinean unos tras otros en filas de dos.

No obstante, es importante introducir un matiz entre estas conclusiones. Y es que el ejemplo extraído del estudio de caso no debe conducir a desdeñar el examen de los espacios de lectura vacíos. Acontecimientos de esta naturaleza recogidos en el cuaderno de campo recuerdan la importancia de emprender el análisis de los espacios físicos (observaciones en la biblioteca escolar, en la biblioteca de pasillo, en el aula del rincón del cómic, etc.) sin descuidar la dimensión que los compromete como espacios letrados: las interacciones que los sujetos producen en su interior (observaciones del taller de relajación para la lectura, de sesiones de trabajo y lectura en el aula de apoyo, de lecturas colectiva en la biblioteca, etc.) 


\section{Observaciones de espacios letrados/ eventos letrados}

a) Observaciones de sesiones de lectura colectiva en la biblioteca

b) Observaciones de las sesiones del taller de relajación para la lectura

c) Observaciones de sesiones de lectura en el aula

d) Observaciones de sesiones de trabajo y lectura en el aula de apoyo

e) Observaciones de sesiones de lectura individual en el aula del rincón del cómic

f) Observaciones en una sesión de reunión de padres

g) Observaciones en una sesión de taller de padres

Observaciones de espacios físicos

h) Observaciones de las producciones pictóricas de los alumnos en los pasillos

i) Observaciones de componentes del aula del rincón del cómic

j) Observaciones de componentes de la Biblioteca Escolar

k) Observaciones en la biblioteca de pasillo

l) Observaciones en el rincón de los poetas

m) Observaciones de carteles y póster sobre lectura colgados en corchos en los pasillos del centro escolar.

Tabla I. Observaciones de espacios físicos y de espacios letrados en la escuela María Moliner.

\section{I.3. El entorno relacional inmediato: hacia un enfoque ecológico de la lectu- ra escolar}

En el caso de la escuela María Moliner, el examen de los espacios letrados y de los eventos letrados se antojaba insuficiente en el trazado del entramado de la lectura.

A juzgar por las observaciones realizadas en el interior de la escuela, la lectura había sido una inquietud constante. La figura de un asno que recordaba a Platero en el recodo de un pasillo, un banco en el jardín frente al patio de recreo y una placa a su lado invitando a la lectura, dibujos del alumnado conmemorativos de la semana cultural fijados en la pared de un pasillo, la existencia de la revista escolar y de una biblioteca con una tradición prolongada, etc. Todos estos elementos letrados constituyen un testimonio de la historia de la lectura y sus prácticas, consolidada en el centro educativo. De hecho, de haber un rasgo por encima de los demás que identifique a la escuela María Moliner ese 
es la pervivencia de su pasado, -tardío o inmediato y bajo la forma de un objeto o de una práctica que perdura-, en su contemporaneidad más reciente. En ciertas ocasiones persevera la memoria de su origen - "Sea lo que sea, lo importante es que leas"-, reza un cartel elaborado por un alumno de quinto curso de Educación Primaria”-, mientras en otras, se ha borrado su recuerdo - "Si quieres aprender, tienes que leer"-, dice otro de aquellos carteles que cuelgan en la pared de uno de los pasillos y cuya autoría, en este caso, se ha olvidado.

La clave de estas pervivencias letradas hay que buscarla en la dimensión humana y micropolítica de la escuela. Junto al profesorado incorporado en los últimos diez años a la plantilla del centro, pertenecen al claustro docentes que llegaron allí entre la década de los setenta y los ochenta, durante los primeros años de la escuela. Algunos de ellos han vivido en el barrio desde su edificación e incluso hay quienes, tras su jubilación, han mantenido una colaboración estrecha y desinteresada con la escuela, quedando al cargo del huerto escolar o del taller de relajación para la lectura.

Sin embargo, los lazos entre el pretérito y el presente de la escuela no se tienden únicamente a través de estos docentes. En el encuentro previo al primer taller de familias que se celebró en la escuela María Moliner, ciertas madres sostenían que su experiencia como alumnas del centro y vecinas del barrio había motivado la matriculación de sus hijos e hijas en la escuela. El mismo parecer fue recogido en la entrevista realizada a un grupo de cuatro mujeres, miembros de la Asociación de Madres y Padres de Alumnos de la escuela.

En todas estas voces testimoniales de docentes y de familias resuenan las reivindicaciones por unas condiciones de vida digna de un barrio obrero al comienzo de la Democracia y de una escuela con algunos sectores de su comunidad educativa próximos a los ideales de Rosa Sensat y a los movimientos de Renovación Pedagógica. En el caso de las madres de familia entrevistadas, dichas reivindicaciones forman parte de una memoria de infancia cuyos ecos alcanzan el presente al proyectar expectativas sobre una nueva generación de alumnos y alumnas: la de sus hijos e hijas. Y en esa memoria de reivindicación y renovación, la lectura aparece entreverada con el lugar de la experiencia como una herramienta para la transformación y el cambio ${ }^{4}$, como un instrumento de empoderamiento para la comunidad o, adoptando un verso de Gabriel Celaya, como "un arma cargada de futuro expansivo" 5 .

En este marco social y político se hacía necesario profundizar en terrenos que no mantenían una relación directa con la lectura. Ese fue el punto de inflexión del estudio que hizo plantear que, quizás, cabría pensar que una investigación etnográfica de estas características, que se aproxima conceptualmente a la lectura como "objeto" socialmente

\footnotetext{
${ }^{4}$ Alguna madre que estudió durante su infancia en otra escuela ha manifestado una visión similar de la lectura, coincidiendo con que en el centro donde fue alumna adoptó un enfoque pedagógico renovador. Sin embargo, en estos otros casos, la decisión de matricular a sus hijos en este centro se sustenta en criterios diferentes como la diversidad del alumnado o la proximidad de la escuela al domicilio.

5 En el original, el verso de Celaya se refiere a la poesía.
} 
situado (Barton y Hamilton, I998), que se interroga tanto sobre la producción de prácticas y discursos sobre la lectura en el seno de una comunidad, como sobre el impacto que tiene en ella y en la forma en que sus miembros se comprenden a sí mismos y a los otros, requiere el análisis del complejo microcosmos en el que la lectura se produce y se transforma. Un microcosmos que compromete a la escuela donde se producen estas prácticas lectoras pero que, al mismo tiempo, rebasa sus propias fronteras, e incluso quiebra el concepto local de barrio y lo trasciende. Un microcosmos cuyas fronteras difusas son definidas por el propio objeto de estudio: la acción de leer y la lectura. Eso es lo que se puede llamar el entorno relacional inmediato o los entornos de proximidad de la lectura (Martos Núñez, 20I3).

El concepto de entorno relacional inmediato bebe directamente de las fuentes de las aportaciones de la corografía y de los estudios de proximidad al análisis de las literacidades (Martos García, 2OIO; Martos Nuñez, 2OI3 y Martos García 2OI4). Frente al topos que designa el territorio físico, el concepto kora, del que proviene el término corografía, se refiere al territorio de la experiencia.

La idea de corografía viene de kora, que es una noción antigua de lugar, distinta al topos.

Kora es el lugar de la experiencia, el barrio o la aldea, a diferencia del topos. Por tanto, la zona a acotar no es una división administrativa sino una «unidad de experiencia» (Martos García, 2014: 153)

Desde esta perspectiva, la corografía posibilita la reconstrucción de "un retrato integrador de los paisajes, prácticas y memoria de la comunidad” (Martos García, 2OIO: 202) donde, deslizando sus aportaciones hasta el análisis de las literacidades, la lectura se encarna y cobra vida en sus múltiples formas.

¿De qué manera se procedió a la hora de recrear el entorno relacional inmediato de la lectura en la escuela María Moliner?, ¿cómo se acota esa unidad de experiencia compartida donde la lectura destaca como una pieza insustituible que ejerce una evidente función de "pegamento social” (Martos García, 2OI4)? Sin duda, existe cierta analogía entre este proceso y lo que tradicionalmente ha sido el análisis contextual de un centro educativo. En ambos casos, se trata de profundizar en las variables sociales y culturales que circundan, que atraviesan y que, en definitiva, calan en una escuela específica. Puede incluir, además, y de hecho en ocasiones es esencialmente necesario, información relevante acerca de la historia tanto del centro educativo como del barrio en que se sitúa. Pero existe, sin embargo, una diferencia fundamental entre un análisis y otro: su origen o punto de partida. Mientras que en el estudio del contexto el epicentro del análisis se sitúa en el propio centro educativo, en el caso de la aproximación al entorno relacional inmediato la mirada etnográfica se debe detener en las prácticas de la lectura escolar y partir de ese punto en la reconstrucción de su contexto natural de producción y dotación de sentido.

Hay, así, una serie de características que definen el procedimiento de elaboración del entorno relacional inmediato de las prácticas contemporáneas de lectura en la escuela María Moliner. 
En primer lugar, el punto de partida del análisis y el lugar en el que el mismo se detiene se establece en las propias prácticas y representaciones de la lectura. Se trata de un proceso de deconstrucción en el que la información que se obtiene a través de las distintas estrategias metodológicas como la observación o la entrevista etnográfica, se descompone en sus elementos sociales y culturales más simples, para proceder posteriormente al rastreo de su origen en el entorno social próximo y al análisis de las causas de su pervivencia en el presente de la escuela.

En segundo lugar, puede suceder que el entramado de la lectura escolar se caracterice por cierta heterogeneidad discursiva. Puede llegar a ocurrir que no se hallen puntos de intersección entre los diferentes discursos que dan forma a la lectura; que, simplemente, coexistan en la escuela compartiendo su tejido simbólico; o, también, puede suceder que estos discursos entren en conflicto y contradicción. La dispersión discursiva no resta valor al entramado de la lectura que la investigación traza, pues la metáfora de dicho entramado no es la imagen de un puzle donde las piezas se ligan dando lugar a una imagen final compacta. Por el contrario, cuando los discursos no se encuentran entre sí, forzar la búsqueda de una lógica subyacente que los mantenga unidos y ofrezca una visión holística de la lectura escolar es un riesgo que puede menoscabar la investigación. Sí es importante tener en cuenta una consecuencia para el análisis del entorno relacional inmediato que se deriva de la heterogeneidad de los discursos: la apertura de múltiples líneas de exploración. De nuevo, el caso de la escuela María Moliner ofrece un ejemplo.

A mediados de los años setenta del siglo XX, la lectura y la escritura en el contexto próximo de este centro educativo habían servido como elemento de cohesión social y empoderamiento comunitario. Una década más tarde, y en un solar baldío del barrio, se instalaron las primeras familias gitanas, constituyéndose el germen de lo que sería el asentamiento chabolista del barrio. Este fue el origen de una nueva red de prácticas y representaciones de la lectura en el imaginario colectivo del barrio y de la escuela, donde las actuaciones autonómicas municipales y locales para la escolarización y la alfabetización del colectivo menor gitano recibían diferentes grados de aceptación y recepción. Ambos acontecimientos mantienen ciertas reminiscencias en la contemporaneidad de la escuela, encarnadas en discursos diferentes acerca de los propósitos de la lectura. Por ese motivo, estos acontecimientos supusieron dos líneas de investigación independientes en la aproximación al entorno relacional inmediato.

En tercer lugar, la delimitación del entorno relacional inmediato supera las fronteras físicas del barrio. En el caso de la escuela María Moliner, algunos de los enclaves que reverberan en la memoria de la comunidad y que constituyen su historia y, en definitiva, que comprenden esa unidad de experiencia que nos aproxima al entramado de la lectura, no forman parte del barrio. O, si lo forman, son compartidos a su vez con otros barrios limítrofes. Ese es el caso, por ejemplo, del parque de Miraflores, cuyo actual estado se debe a la acción vecinal conjunta del barrio donde se sitúa la escuela y de otros barrios colindantes. El territorio comprendido finalmente en el proceso de la investigación se extendía a lo largo de nueve barrios de la periferia norte de la ciudad de Sevilla. 
En cuarto lugar, se trata de un análisis que implica un cierto esfuerzo multidisciplinar, que precisa de las aportaciones de la Sociología, la Geografía o la Historia, entre otras disciplinas y que se sustenta sobre una compleja miríada de fuentes y estrategias metodológicas.

\section{Las estrategias metodológicas de recogida de la información}

Dentro del proyecto de la investigación, la recogida de información fue estipulada para efectuarse a través de algunas de las estrategias metodológicas ortodoxas en la tradición etnográfica educativa. Las entrevistas, las conversaciones informales, el análisis de documentos y la observación etnográfica se contemplaban entre las estrategias preferentes, mientras que el cuaderno de campo, el diario de la investigación y el cronograma se establecieron, siguiendo las recomendaciones de Vázquez Recio (2OII) como recursos y soportes para la recogida de la información. Sin embargo, el propio trabajo de campo y el curso de la investigación exigieron la búsqueda de estrategias alternativas igualmente significativas para la etnografía de la lectura escolar, como fueron los casos de la fototeca municipal de Sevilla, el fondo de la hemeroteca del periódico ABC, la consulta de algunos mapas y planos de la capital hispalense de los siglos XVIII al XX, o la revisión bibliográfica de aquellas obras que se habían ocupado de algún que otro aspecto relacionado con la historia del centro educativo y de su entorno y que prestaban ayuda en la reconstrucción del entramado de la lectura escolar. De ese modo, el recabado de los datos se transformó en un proceso flexible y abierto cuyas estrategias metodológicas iban modulándose y en función de las cuestiones de indagación emergentes. Lo que sigue a continuación es un análisis sucinto acompañado de algunas reflexiones acerca de las aportaciones a la investigación de todas estas estrategias de recogida de la información.

a) La entrevista etnográfica:

Stake dice a propósito de la entrevista etnográfica que:

Mucho de lo que no podemos observar personalmente, otros lo han observado o lo están observando. Dos de las utilidades principales del estudio de casos son las descripciones y las interpretaciones que se obtienen de otras personas. No todos verán el caso de la misma forma. Los investigadores cualitativos se enorgullecen de descubrir y reflejar las múltiples visiones del caso. La entrevista es el cauce principal para llegar a las realidades múltiples (2007: 63).

Las entrevistas etnográficas fueron una pieza clave en la aproximación a las representaciones de la lectura en el interior de la comunidad educativa de la escuela María Moliner. Las entrevistas permiten ahondar en las observaciones realizadas en las sesiones de campo (Balcázar, 2005), pues a través de la "palabra ofrecida” (Vázquez Recio, 2OII) 
se le presenta al sujeto que investiga no sólo la descripción, sino también el sentido que lo observado adquiere para un individuo o una comunidad concreta, $y$, por tanto comprender el proceso de construcción de las realidades letradas escolares mediante el acercamiento holístico a sus prácticas.

En otras ocasiones, las entrevistas etnográficas arrojaron luz sobre objetos, prácticas, costumbres y ritos de la vida cotidiana escolar y, en definitiva, sobre espacios y eventos letrados a los que la mirada investigadora no alcanzaba; bien por mediar una distancia espacial entre ambos, en el caso de estar ausente del lugar del acontecimiento, bien por mantener un distanciamiento temporal al tratarse de un acontecimiento ya pasado. En ambas situaciones, la entrevista encontraba su valor como fuente oral de un conocimiento inaccesible a la mirada investigadora.

En el estudio de caso efectuado, las entrevistas tendieron un puente hacia la historia de las prácticas y representaciones de la lectura en el centro escolar y su relación con su entorno próximo, permitiendo reconstruir su entorno relacional inmediato. Una historia para la que la mayor parte de las fuentes documentales escritas habían ido desapareciendo con los años de los archivos de la escuela. En ese caso, el grueso de información vino de la mano de los docentes de mayor arraigo y pertenencia en el centro escolar, algunos ya jubilados; la mayoría, vecinos del propio barrio o de barriadas limítrofes. Pero incluso cuando el examen de las fuentes documentales escritas o los períodos de observación etnográfica arrojaron información importante para el estudio, la entrevista resultó ser una estrategia metodológica fundamental para complementar la información recabada.

\section{b) La observación etnográfica:}

En la aproximación analítica a la escuela María Moliner, la observación etnográfica de prácticas de lectura y espacios letrados cumplía dos funciones elementales en el proceso de recogida de los datos.

Por una parte, la observación etnográfica aportaba a la investigación un nuevo prisma de la información obtenida a través de otras estrategias metodológicas, principalmente de las entrevistas y de las conversaciones informales, permitiendo a posteriori integrar el conjunto de datos recabados dentro de un contexto global de sentido. En esa línea, Massot, Dorio y Sabariego dicen a propósito de la observación etnográfica que "permite observar la y realidad social en su conjunto, desde una perspectiva holística" (2004: 333). Por otro lado, la observación etnográfica ofrecía nuevos ámbitos de exploración del entramado de la lectura en la escuela que tras las entrevistas podían haber pasado desapercibidos o permanecido en penumbra, porque formaban parte de los valores, creencias y normas interiorizados por la comunidad escolar (Balcázar, 2005). De ese modo, la observación etnográfica brindaba nuevos ámbitos sobre los que volver y profundizar con mayor detenimiento. Es decir, durante la estancia en el campo, la observación etnográfica y las entrevistas mantuvieron una relación dialéctica y de retroalimentación. 
c) Las conversaciones informales:

La entrevista informal puede variar desde discusiones causales mientras se participa en una actividad, a entrevistas abiertas o discusiones en profundidad con informantes clave (Latorre, Del Rincón y Arnal, 2005: 230).

La entrevista informal es prácticamente consustancial a una realidad social donde se producen continuos encuentros entre individuos. En ocasiones, las conversaciones informales surgían en el período entre clases, entre dos docentes que se encontraban sin buscarse y comentaban los percances de la jornada con un alumno; en la sala de profesores, donde surgía el tema de la decreciente matrícula del alumnado o del incremento de absentismo escolar los Viernes, días de mercadeo ambulante; en la biblioteca, donde un docente preguntaba sobre cómo sería la próxima colaboración en la revista escolar; en el patio de recreo... y en cualquier otro espacio del centro donde la conversación se iniciaba espontáneamente, sin un guión elaborado previamente..

¿Cuál es entonces el lugar del investigador o investigadora en las conversaciones informales o en situaciones no construidas? El cometido principal ha de ser prestar atención a los temas que surgen de manera imprevista, pues las conversaciones informales no sólo son útiles en sí mismas, en el sentido de que aportan información sustancial. Sirven también para crear nuevos ámbitos de exploración en las entrevistas etnográficas y nuevos ángulos para la observación.

\section{d) Fuentes documentales escritas:}

Un texto escrito es un testimonio mudo que permanece físicamente, conserva su contenido a lo largo del tiempo y con él, sin embargo se puede efectuar una entrevista. No se puede hablar directamente con él, pero puede ser interpretado (Ruiz Olabuénaga, 2OI2: 193).

El análisis de documentos es otra de las estrategias clave para la recogida de información, pues, parafraseando a Ruiz Olabuénaga, "es un texto mudo que permanece físicamente" (2OI2:I93). El sujeto que investiga no puede mantener una relación dialógica con él, pero puede acceder a su información escrita.

En el proceso de análisis documental se examinó un volumen importante de documentos que, atendiendo a su finalidad, se clasificaban en dos grupos. Por un lado, estaban las fuentes documentales que sirvieron para reconstruir el entorno relacional inmediato de la lectura, analizando en la escuela y en su entorno cercano la aparición del interés hacia las prácticas de lectura y hacia la biblioteca escolar. En segundo lugar, estaban aquellas otras fuentes documentales que permitían comprender las prácticas y representaciones de la lectura actual.

Del centro se obtuvieron una cantidad importante de documentos internos; documentos que pueden ordenarse en dos grupos: aquellos de carácter formal, que forman parte de la gestión y de la organización del centro; y aquellos otros de naturaleza informal que se crean el transcurso de la cotidianidad escolar. 
Entre los documentos formales del centro escolar se hallarían la Memoria Final del Curso 2009/20Iо, el Plan Anual de Centro 20Iо/20II, el Plan de Convivencia, el Plan de Acción Tutorial, el Plan de Atención a la Diversidad y el más importante en este caso, el Plan de Lectura y Biblioteca para el curso escolar 2OIO/2OII, que define objetivos y actividades para la lectura dentro de la escuela y para su biblioteca escolar.

Por otra parte, los documentos informales fueron un elemento relevante en el análisis documental. Este tipo de documentos, de índole diversa, se consiguió principalmente de la biblioteca: el proyecto de creación de la revista escolar, anotaciones sobre su proceso creativo, documentos para su evaluación, ejemplares de la misma revista, libros producidos por los alumnos e instrucciones para el profesorado y el alumnado. Igualmente, se debe incluir aquí un libro que me fue prestado sobre la historia del barrio y del centro escolar y cuyo título no se puedo revelar sin dar conocer la identidad del barrio y de la escuela donde se realizó el estudio de caso, rompiendo así uno de los principios éticos de la investigación: el anonimato. El Libro fue escrito por vecinos del barrio "para que su memoria no se perdiese" y constituye, una de las fuentes documentales más interesantes, al recoger extractos de testimonios de sus protagonistas.

\section{e) El recurso de la hemeroteca:}

La consulta de la hemeroteca no deja de ser un parte de las fuentes documentales escritas. Sin embargo, al ser una fuente de información poco convencional en las investigaciones etnográficas educativas, recibe aquí un tratamiento especial y diferenciado.

Los archivos de la prensa sevillana examinados se correspondían principalmente con el período comprendido entre los años I972-I982, lo que constituyó la primera década de funcionamiento del centro. Los artículos consultados hacían referencia a la implantación del Plan de Urgencia para Andalucía (PUA), una medida desarrollada en el contexto de la Ley General de Educación de I97O para paliar los déficits de plazas escolares. La escuela se construyó bajo la iniciativa del PUA, y su infraestructura respondía a un modelo pedagógico concreto que otorgaba un marco particular a la lectura en la escuela. Este campo ha sido superficialmente tratado en la bibliografía pedagógica, de modo que la prensa escrita resultó ser una fuente de información útil, rica y valiosa en la reconstrucción del entorno relacional inmediato del entramado de la lectura en la escuela María Moliner.

\section{f) El fondo de la Fototeca Municipal de Sevilla}

Las imágenes del archivo histórico de la fototeca municipal de Sevilla constituyeron una referencia de primer orden de las transformaciones del contexto sociocultural del centro escolar, con el fin de conocer sus raíces, comprenderlas sus y discernir sobre las posibles relaciones de la escuela con su entorno próximo, el barrio. 


\section{g) Cartografía: mapas y planos de la ciudad de Sevilla}

Las referencias al entorno y su historia surgían espontáneamente en las conversaciones informales o en las entrevistas realizadas lo que hacía necesario conocer el origen de aquellas referencias y valorar su peso en la comprensión de las prácticas letradas. Recorrer las calles del barrio y sus contornos, anotando nombres de calles y cotejando esos espacios con planos de la ciudad de los siglos XVIII al XX fue de gran ayuda para profundizar en la comprensión de la información suministrada por los informantes, y evitar así desplazar la entrevista con interrupciones aclarativas ${ }^{6}$.

\section{Conclusiones: etnografía, lectura y escuela}

La comprensión de la lectura como práctica social situada y contingente propiciada por los Nuevos Estudios de Literacidad, ha dejado al descubierto líneas de investigación emergentes en el ámbito de la educación que se sitúan más allá de los tradicionales enfoques psicológico y lingüístico. Ante esta perspectiva, la etnografía educativa hace frente a nuevos desafíos. En este artículo se han explorado tres posibles ámbitos de indagación etnográfica: por una parte, los usos de la lectura, las representaciones letradas y las identidades (Martos García, 2OIO); por otra, los espacios letrados, y, finalmente, el entorno relacional inmediato o los entornos de proximidad de la lectura (Martos Núñez, 20I3). Para ilustrar cada uno de estos ámbitos se ha tomado como referencia un estudio de caso realizado en la ciudad de Sevilla y cuyo objetivo era el análisis de las prácticas, las representaciones y los discursos que sobre la lectura se generaban en un centro de Educación Infantil y Primaria.

Algunas de las conclusiones extraídas de este estudio y revisadas en este artículo son las que se exponen a continuación.

Así y en primer lugar, en el contexto de la escuela las cuestiones acerca de los usos de la lectura y de las prácticas letradas pueden formularse mediante el recurso de la entrevista a los propios lectores: el alumnado (Aliagas, 2008; Aliagas, Cassany y Castellá, 2009). Sin embargo, es igualmente preciso indagar en las representaciones existentes entre el profesorado, las familias y otros agentes de la comunidad educativa acerca de los límites que identifican a los buenos lectores, los propósitos de la lectura, el sentido de las prácticas letradas y de la animación a la lectura, etc.

En segundo lugar, el examen de las representaciones letradas escolares plantea, al menos, una dificultad. La naturalización de la lectura dentro de las organizaciones escolares la hace surgir como un fenómeno atemporal, descontextualizado y universal, un

\footnotetext{
${ }^{6}$ El libro donde se hallaron los planos necesarios fue Cortés, J.; García Jaén, M. J. y Zoido, F. (I992). Planos de Sevilla. Colección Histórica. 1771-1918. Sevilla, Servicio de Publicaciones del Ayuntamiento de Sevilla. La obra puede consultarse en el Archivo Histórico de la página web de la Gerencia de Urbanismo del Ayuntamiento de Sevilla. Recientemente, se han integrado en esta plataforma fotografías aéreas de la ciudad de Sevilla realizadas en los últimos quince años. Véase: http://www.sevilla.org/urbanismo/
} 
hecho que obstaculiza el acceso a las representaciones de la lectura y a su incardinación dentro de unas coordenadas sociales, culturales y educativas concretas. La problematización de la lectura, su "desacralización" mediante el proceder etnográfico requiere de la utilización de estrategias metodológicas de recogida de la información diversas (las entrevistas, la observación, el análisis de documentos, las conversaciones informales, etc.) que permitan enfocar las mismas cuestiones desde diferentes ángulos.

En tercer lugar, se ha señalado la relevancia de examinar los espacios físicos destinados a la lectura como escenarios de lectura en acción. Los períodos de observación de los espacios letrados escolares vacíos (la biblioteca de centro o el rincón del cómic, por citar dos ejemplos), deben seguirse de períodos de observación de estos mismos espacios cuando en su interior se desarrollan prácticas letradas (lecturas colectivas o creación y lecturas de cómics, siguiendo con el ejemplo anterior).

En cuarto y último lugar se ha hecho referencia al contexto relacional inmediato o los entornos de proximidad de la lectura, conceptos que fueron muy útiles en el análisis del caso de la escuela María Moliner. La experiencia de la lectura en este centro educativo traspasaba sus fronteras y solo podía ser comprehendida con profundidad contemplándola desde el sentido que adquiría en el barrio y sus proximidades. Este espacio experiencial del que forma parte la escuela por mediación de las prácticas y las representaciones letradas compartidas es el entorno relacional inmediato. 


\section{Bibliografía}

- Aliagas, C. (2008). Las prácticas lectoras adolescentes: cómo se construye el desinterés por la lectura. En Actas del VIII Congreso de Lingüística General 20o8. Recuperado el 4 de Noviembre de 20I5 de http://elvira.lllf.uam.es/clg8/actas/pdf/paperCLG5.pdf

- Aliagas, C.; Castellà, J. M. y Cassany, D. (2009). "Aunque lea poco, yo sé que soy listo". Estudio de caso sobre un adolescente que no lee literatura. Revista OCNOS, nº 9, 97-II2.

- Balcázar Nava, P.; González Arratia, N. I.; Gurriola Peña, G. y Moysén Chimal, A. (2005). Investigación cualitativa. Toluca: Universidad Autónoma del Estado de México.

- Barton, D. y Hamilton, M. (1998). La literacidad entendida como práctica social. En V. Zavala; M. Niño-Murcia y P. Ames (Eds.). Escritura y sociedad. Nuevas perspectivas teóricas y etnográficas (pp. Io9-I39). Lima: Red para el Desarrollo de las Ciencias Sociales en el Perú, 2004 .

- Cassany, D. (2006). Tras las líneas. Sobre la lectura contemporánea. Barcelona: Anagrama.

- Chartier, R. (1994). Elorden de los libros: lectores, autores, bibliotecas en Europa entre los siglos XIV y XVIII. Barcelona: Gedisa.

- Cortés, J.; García Jaén, M. J. y Zoido, F. (1992). Planos de Sevilla. Colección Histórica. 17711918. Sevilla: Servicio de Publicaciones del Ayuntamiento de Sevilla

- Díaz de Rada, A. (2012). El taller del etnógrafo. Materiales y herramientas de investigación en etnografía. Madrid: Ediciones UNED.

- Gimeno Sacristán, J. (2003). El alumno como invención. Madrid: Morata.

- González Landa, M. C. (2009). Aproximación al leer y al escribir como procesos interactivos y situados. Didáctica. Lengua y Literatura, vol. 2I, I57-I9O.

- Latorre Beltrán, A.; Del Rincón Igea, D. y Arnal Agustín, J. (2005). Bases metodológicas de la investigación educativa. Barcelona: Ediciones Experiencia.

- Martos García, A. (2013). Eventos letrados. En E. Martos Núñez y M. Campos (Coords.). Diccionario de nuevas formas de lectura y escritura (pp. 247-250). Madrid: Red Internacional de Universidades Lectoras-Santillana.

- Martos García, A. E. (2OIO). Las prácticas de lectura/ escritura y los enfoques etnográfico y geográfico. Didáctica. Lengua y Literatura, vol. 29, I99-229. 
- Martos García, A. E. (20I4). Aportaciones de la geografía y la cartografía a los estudios de literacidad. En M. Campos y E. Martos Núñez (Coords.). Cartografías lectoras y otros estudios de lectura. Lectura en las universidades públicas andaluzas (pp. I45-I55). Madrid: Red Internacional de Universidades Lectoras-Marcial Pons.

- Martos Núñez, E. y Vivas Moreno, A. (Coords) (2OIO). Performance, lectura y escritura (Conceptos y prácticas). Madrid: Universidad Complutense, Red Internacional de Universidades Lectoras, Colección Temas de Lectura.

- Martos Núñez, E. y Martos García, A. E. (2OI2). De los espacios de lectura a los espacios letrados. Pulso, nº35, Io9-I29.

- Martos Núñez, E. (20I3). Entornos de proximidad. Corografía. En E. Martos Núñez y M. Campos (Coords.) Diccionario de nuevas formas de lectura y escritura (pp. 2I5-2I7). Madrid: Red Internacional de Universidades Lectoras-Santillana.

- Martos Núñez, E. (20I3b). Espacios letrados. En E. Martos Núñez y M. Campos (Coords.) Diccionario de nuevas formas de lectura y escritura (pp. 234-236). Madrid: Red Internacional de Universidades Lectoras-Santillana.

- Moreno Bayona, V. (2005). Lectores competentes. Revista de Educación, $n^{\circ}$ extraordinario, $\mathrm{I} 53-\mathrm{I} 67$.

- Massot Lafon, M. I.; Dorio Alcaraz, I. y Sabariego Puig, M. (2004). Estrategias de recogida y análisis de la información. En R. Bisquerra Alcina (Coord.). Metodología de la investigación educativa (pp. 329-366). Madrid: La Muralla.

- Ruiz Olabuénaga, J. I. (2OI2). Metodología de la investigación cualitativa. Bilbao: Deusto. Serie Ciencias Sociales, vol. I5.

- Stake, R. (2007). Investigación con estudio de casos. Madrid: Morata.

- Vázquez Recio, R. (2002). La dirección de centro y sus metáforas: símbolo, acción y ética. Estudio de un caso. Universidad de Cádiz (Tesis doctoral inédita).

- Vázquez Recio, R. (2OII). Investigar con estudio de caso la dirección escolar. Málaga: Aljibe.

- Viñao Frago, A. (1999). Leer y escribir. Historia de dos prácticas culturales. Naucalpán de Juárez: Fundación Educación, Voces y Vuelos.

- Viñao Frago, A. (2002). La enseñanza de la lectura y la escritura: análisis sociohistórico. Anales de Documentación, $\mathrm{n}^{\circ}$ 5, 345-359. 